\title{
Interpersonal synergies
}

\section{Michael A. Riley ${ }^{*}$, Michael J. Richardson ${ }^{1}$, Kevin Shockley ${ }^{1}$ and Verónica C. Ramenzoni²}

1 Center for Cognition, Action, and Perception, Department of Psychology, University of Cincinnati, Cincinnati, OH, USA

2 Communication Before Language, Max Planck Institute, Nijmegen, Netherlands

\section{Edited by:}

Carolee Winstein, University of

Southern California, USA

\section{Reviewed by:}

Richard Schmidt, College of the Holy Cross, USA

Linda Fetters, University of Southern California, USA

John Scholz, University of Delaware, USA

\section{*Correspondence:}

Michael A. Riley, Center for Cognition, Action, and Perception, Department of Psychology, University of Cincinnati, Mail Location 0376, Cincinnati, $\mathrm{OH}$ 45221-0376, USA

e-mail:michael.riley@uc.edu
We present the perspective that interpersonal movement coordination results from establishing interpersonal synergies. Interpersonal synergies are higher-order control systems formed by coupling movement system degrees of freedom of two (or more) actors. Characteristic features of synergies identified in studies of intrapersonal coordination - dimensional compression and reciprocal compensation - are revealed in studies of interpersonal coordination that applied the uncontrolled manifold approach and principal component analysis to interpersonal movement tasks. Broader implications of the interpersonal synergy approach for movement science include an expanded notion of mechanism and an emphasis on interaction-dominant dynamics.

Keywords: interpersonal coordination, synergy, cross-recurrence quantification analysis, principal component analysis, joint action
The need to coordinate with others arises often in daily life. Examples include dancing, shaking hands, conversing, and playing team sports. Interpersonal coordination has been extensively studied (e.g., Condon and Ogston, 1967; Kendon, 1970; Schmidt et al., 1990; Bernieri and Rosenthal, 1991; Chartrand and Bargh, 1999; Shockley et al., 2003; Reed et al., 2006; Tognoli et al., 2007). Research on interpersonal coordination may reveal very general principles of motor behavior, and can aid in understanding phenomena as far-ranging as language, communication, and socialization (Marsh et al., 2009; Shockley et al., 2009). We propose (see also Marsh et al., 2006, 2009; Fowler et al., 2008; Latash, 2008; Richardson et al., 2008a, 2010; Schmidt and Richardson, 2008; Shockley et al., 2009) that, like intrapersonal coordination, interpersonal coordination is achieved by linking the degrees of freedom (DF) of the motor system (or two actors' motor systems) into synergies (Gelfand and Tsetlin, 1966; Bernstein, 1967; Turvey et al., 1978)².

\section{DEGREES OF FREEDOM AND SYNERGIES}

Bernstein (1967) identified the degrees of freedom problem - the notion that the large number of independently controllable movement system DF poses a computational burden to the CNS (Turvey et al., 1982; Turvey, 1990). Bernstein's solution (see also Gelfand and Tsetlin, 1966; Turvey et al., 1978; Tuller et al., 1982) was that rather than controlling each DF separately, the DF are coupled to form a synergy, enabling the $\mathrm{DF}$ to regulate each other. This reduces the need to control each DF, and allows compensation for variability in one component of the synergy by another. Two central features of synergies are dimensional compression and reciprocal compensation.

\footnotetext{
${ }^{1}$ Some authors use the term coordinative structures to refer to synergies, but we use synergy for brevity. The terms can be used interchangeably but have distinct emphases - "synergy" often implies a muscular level of analysis, and sometimes describes the collective effects of a group of muscles without implying other subtleties such as reciprocal compensation.
}

\section{DIMENSIONAL COMPRESSION}

Dimensional compression (Kay et al., 1987) is expressed in Figure 1 (Kay, 1988; Riley and Turvey, 2002). DF that potentially are independent are coupled so that the synergy has fewer DF (possesses a lower dimensionality) than the set of components from which it arises. The behavior of the synergy has even fewer DF, a second level of dimensional compression as one moves from structural components to the behavior enacted by the interactions among the DF. Dimensional compression at both stages results from imposing constraints, which couple components so they change together rather than independently.

Dynamical systems approaches have emphasized dimensional compression (Kugler et al., 1980; Kugler and Turvey, 1987; Kay, 1988; Turvey, 1990; Turvey and Carello, 1996). Dimensional compression occurs in self-organization (Nicolis and Prigogine, 1977) and features prominently in synergetics (Haken, 1983), a theory of self-organization that describes how systems of many non-linearly interacting, micro-scale components exhibit lowdimensional spatio-temporal patterns. These patterns are described as order parameters which describe the system's macroscopic order. Interactions among micro-components give rise to the macroscopic pattern, which then constrains the behavior of the micro-components to sustain the pattern.

Coordination dynamics (Kelso, 1995) applies synergetics to describe how micro-scale neuromuscular processes give rise to macroscopic movement patterns. In interlimb rhythmic coordination (synchronized oscillations of body segments, such as the rhythmic patterns of walking legs), the order parameter relative phase (the difference in the segments' oscillation phases) captures the low-dimensional behavior that arises from the high-dimensional neuromuscular system. Relative phase describes the spatiotemporal pattern of rhythmic coordination and the changes in coordination that occur in response to manipulations of control parameters (e.g., movement frequency). The dynamics of relative phase are understood to reflect the behavior of a synergy (Kelso, 1994; Turvey and Carello, 1996). 


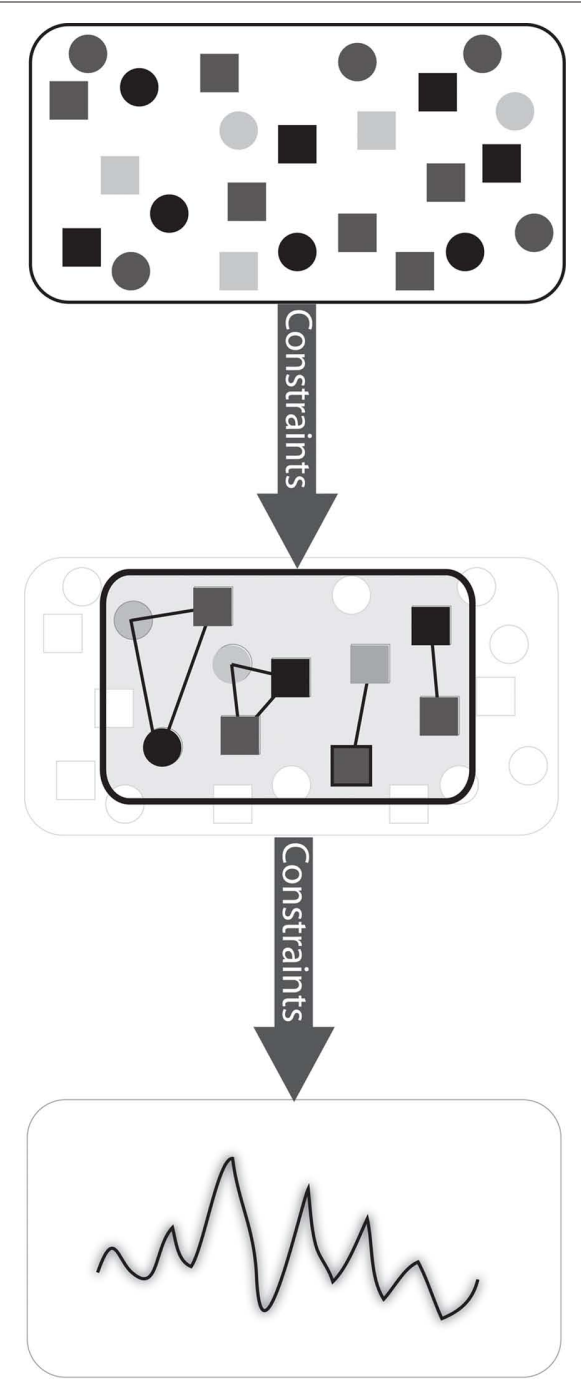

FIGURE 1 | Schematic depiction of a synergy.

\section{RECIPROCAL COMPENSATION}

Dimensional compression is a necessary but insufficient condition for the existence of a synergy (Latash, 2008). The second and more critical characteristic of synergies, reciprocal compensation, refers to the ability of one component of a synergy to react to changes in others. A classic example occurs when one effector is perturbed during speech (Kelso et al., 1984). When the lower jaw was tugged downward, it was quickly compensated by a reciprocal change (the lower lip extended upward) that enabled the speaker to complete pronunciation of the sound.

Reciprocal compensation is central to the uncontrolled manifold (UCM) approach (Scholz and Schöner, 1999; Latash et al., 2002). This approach assumes that coordinated movement is achieved by stabilizing the value of a performance variable (such as a value of relative phase corresponding to an interlimb coordination pattern). In doing so, a subspace (i.e., manifold) is created within a state space of task-relevant elements (the DF that participate in the task, for example the angular positions and velocities of two oscillating limbs), such that within the subspace the value of the performance variable remains constant. ${ }^{2}$ This subspace is called the UCM. Component values that do not lead to desired values of the performance variable (values outside the UCM) are restricted, whereas values that do not affect the performance variable (those within the UCM) are allowed.

An example (Latash et al., 2002) is using two fingers to produce a total force of, for example, $10 \mathrm{~N}$. This target can be achieved by producing $5 \mathrm{~N}$ of force with each finger, or by pressing unequally hard with the fingers but so that the total force is $10 \mathrm{~N}$ (e.g., one finger produces $9 \mathrm{~N}$ and the other produces $1 \mathrm{~N}$, or one produces $7 \mathrm{~N}$ while the other produces $3 \mathrm{~N}$, etc.). If the force produced by each finger is plotted on orthogonal axes, the UCM is a subspace of the resulting plane, a line corresponding to Force $_{\text {finger 1 }}+$ Force $_{\text {finger } 2}=10 \mathrm{~N}$. As long as performance falls along the UCM, the target force is achieved as one finger compensates for the other.

In the UCM analysis the hypothesized stabilization of a performance variable is evaluated by computing two quantities. The first, variance along the UCM, measures the extent to which variability among the DF is compensated to preserve the performance variable. Variance perpendicular to the UCM measures uncompensated variability that causes the performance variable to deviate from the target. While the individual variance components are often informative, their ratio provides a compact measure of the existence and strength of a synergy. If compensated variance is greater than uncompensated variance (ratio $>1$ ), the hypothesized performance variable is stabilized by compensation among the DF - a synergy exists - whereas a ratio $\leq 1$ means the synergy does not exist. The higher the ratio, the greater the amount of compensated variance, suggesting a stronger synergy (depending on the magnitude of uncompensated variance).

\section{INTERPERSONAL SYNERGIES}

Apparent interpersonal coordination could be merely incidental (Figure 2A) - rather than reflecting true coordination, with the signatures of dimensional compression and reciprocal compensation, people may appear to coordinate their movements because they simultaneously execute similar motor programs, mediated by shared motor representations (Garrod and Pickering, 2004, 2009; Sebanz et al., 2006). The studies described below contradict this alternative hypothesis and instead support the interpersonal synergy hypothesis (see Figure 2B). One study applied the UCM analysis to interpersonal rhythmic movement coordination (Black et al., 2007) and another applied principal component analysis (PCA) to performance of an interpersonal precision task (Ramenzoni, 2008).

We focus on Black et al. (2007) and Ramenzoni (2008) because they directly address dimensional compression and reciprocal compensation. There is an extensive literature on interpersonal coordination. For example, Condon and Ogston (1971) hand-scored videotapes of interpersonal interactions to determine when conversants spoke relative to one another. Cohn and Tronick (1988) evaluated when similar affect was exhibited by interacting mothers and children. Newtson et al. $(1977,1987)$ quantified interpersonal

${ }^{2}$ The mapping from a higher-dimensional space of elements to a low-dimensional (usually one-dimensional) space of the performance variable is another manifestation of dimensional compression. 


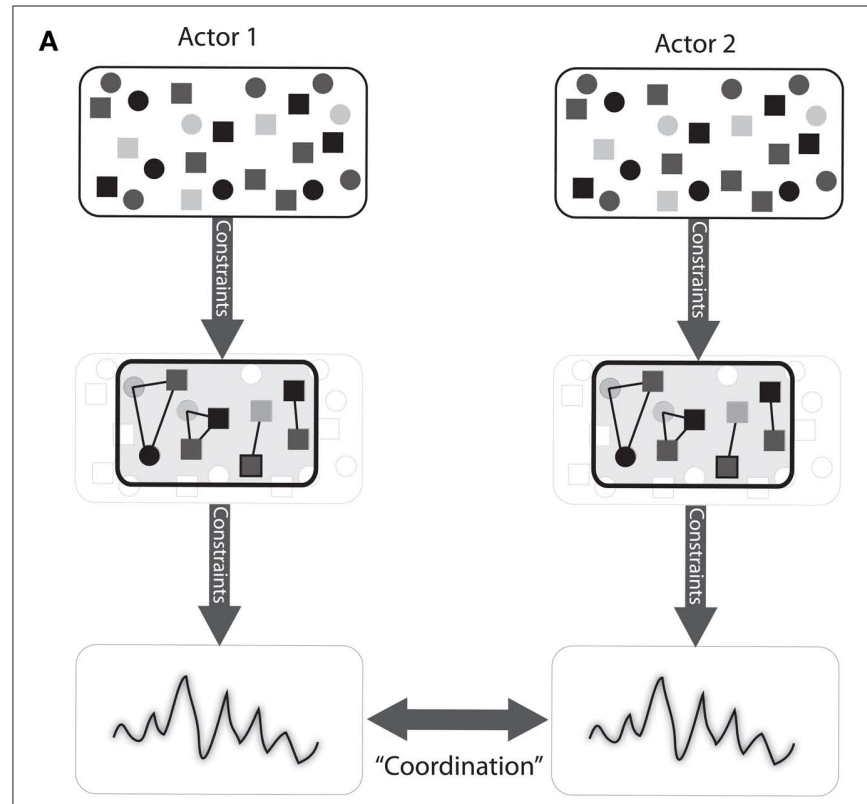

B

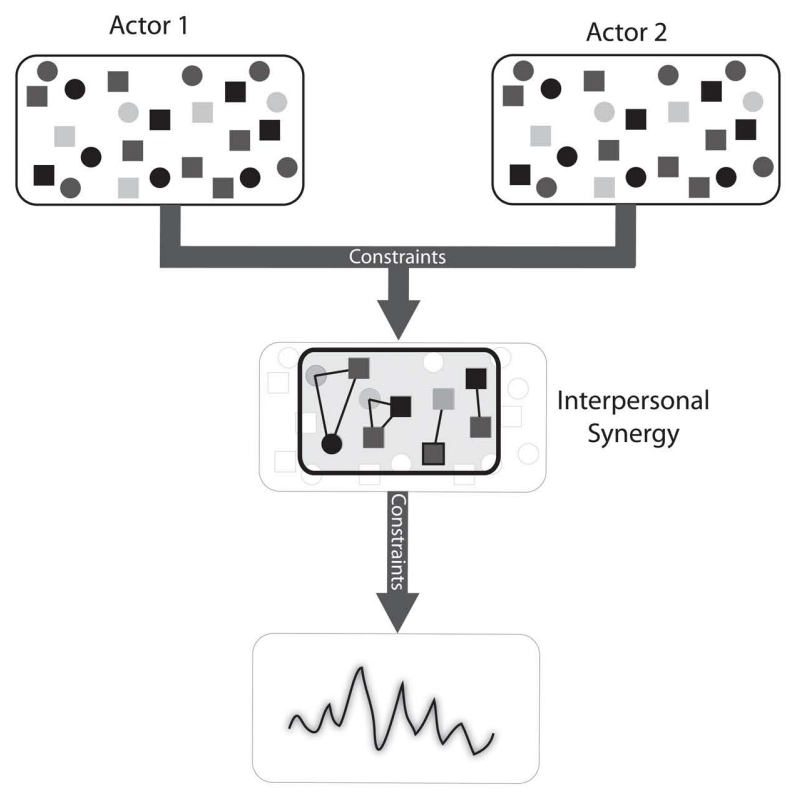

FIGURE 2 | (A) Hypothetical mechanism for appearance of interpersonal coordination. Each actor assembles a synergy that achieves the movement pattern required by the actor's role in the task. Because actors share similar representations of the task they are able to execute similar but independent movement patterns without demonstrating the reciprocal compensation that characterizes synergies. (B) Hypothetical mechanism for interpersonal coordination that involves the formation of a joint or interpersonal synergy, composed of elements of each actor's movement system.

coordination by hand-scoring joint-angle changes in videos of interacting participants. The degree of overall joint-angle change over time was submitted to spectral analysis to capture movement periodicity, and the spectral profiles were compared using coherence analysis to evaluate how similar two "behavioral waves" were. The methodology of these studies does not allow for direct evalua- tion of the interpersonal synergy hypothesis, although the processes of interpersonal coordination they documented is consistent with that hypothesis.

Programmatic studies on interpersonal rhythmic coordination (reviewed by Schmidt and Richardson, 2008) have also produced evidence for interpersonal synergies. Coordination of rhythmically moving limbs between two individuals exhibits the same phenomena observed within individuals and predicted by the HKB model of interlimb rhythmic coordination (Haken et al., 1985) - the existence of two spontaneously stable coordination patterns (in-phase and anti-phase), transitions from anti-phase to in-phase at a critical movement frequency, critical fluctuations (increases in movement variability) preceding the transition, and a shift in the phase relation between oscillating segments when coordinating segments with different intrinsic frequencies (Schmidt et al., 1990; Schmidt and Turvey, 1994). These findings are important because according to the dynamical systems perspective, intrapersonal interlimb rhythmic coordination is a paradigmatic synergy (e.g., Kugler and Turvey, 1987; Turvey, 1990; Kelso, 1995; Turvey and Carello, 1996). This raises the possibility that interpersonal coordination likewise reflects the activity of a synergy supported by visual coupling of the actors' DF (Schmidt and Richardson, 2008; Marsh et al., 2009; Richardson et al., 2010).

\section{INTERPERSONAL MANUAL-POSTURAL SYNERGIES}

The first study we discuss (Ramenzoni, 2008) involved pairs of participants performing an interpersonal precision task. One participant held a target (a circle), while another held a pointer through the circle without touching its sides (Figure 3A). Smaller circles were used to create more difficult task conditions. Preliminary results showed that with greater task difficulty participants' hand and torso movements became increasingly coordinated (as quantified by cross-recurrence quantification analysis - CRQA; Webber and Zbilut, 1994; Shockley et al., 2002; Shockley, 2005; Richardson et al., 2007). CRQA quantifies the degree of shared activity between two time series by evaluating how they unfold similarly over time in a multi-dimensional (embedding) space. The proportion of body configurations shared between the time series (an overall measure of coordination) and how long they maintain similar patterns (how stable the coordination is) are among the CRQA measures (see Webber and Zbilut, 1994).

Displacements of participants' hands, forearms, arms, and torsos were tracked in 3-D as they performed the interpersonal task or performed the subtasks (pointing, holding the target) without interacting with each other in an individual-task condition. PCA was used to identify dimensional compression. PCA identifies relations within high-dimensional datasets and maps the original data into a space whose axes (principal components) represent the dataset's primary dimensions of variation (Daffertshofer et al., 2004; Forner-Cordero et al., 2005). Those dimensions can be abstract and need not map directly onto the original dataset's dimensions. If the original variables are correlated, PCA yields dimensional reduction - fewer dimensions are required to account for most of the variation in the dataset (a criterion of $90 \%$ of the variance was employed in this study) than the number of original variables. 

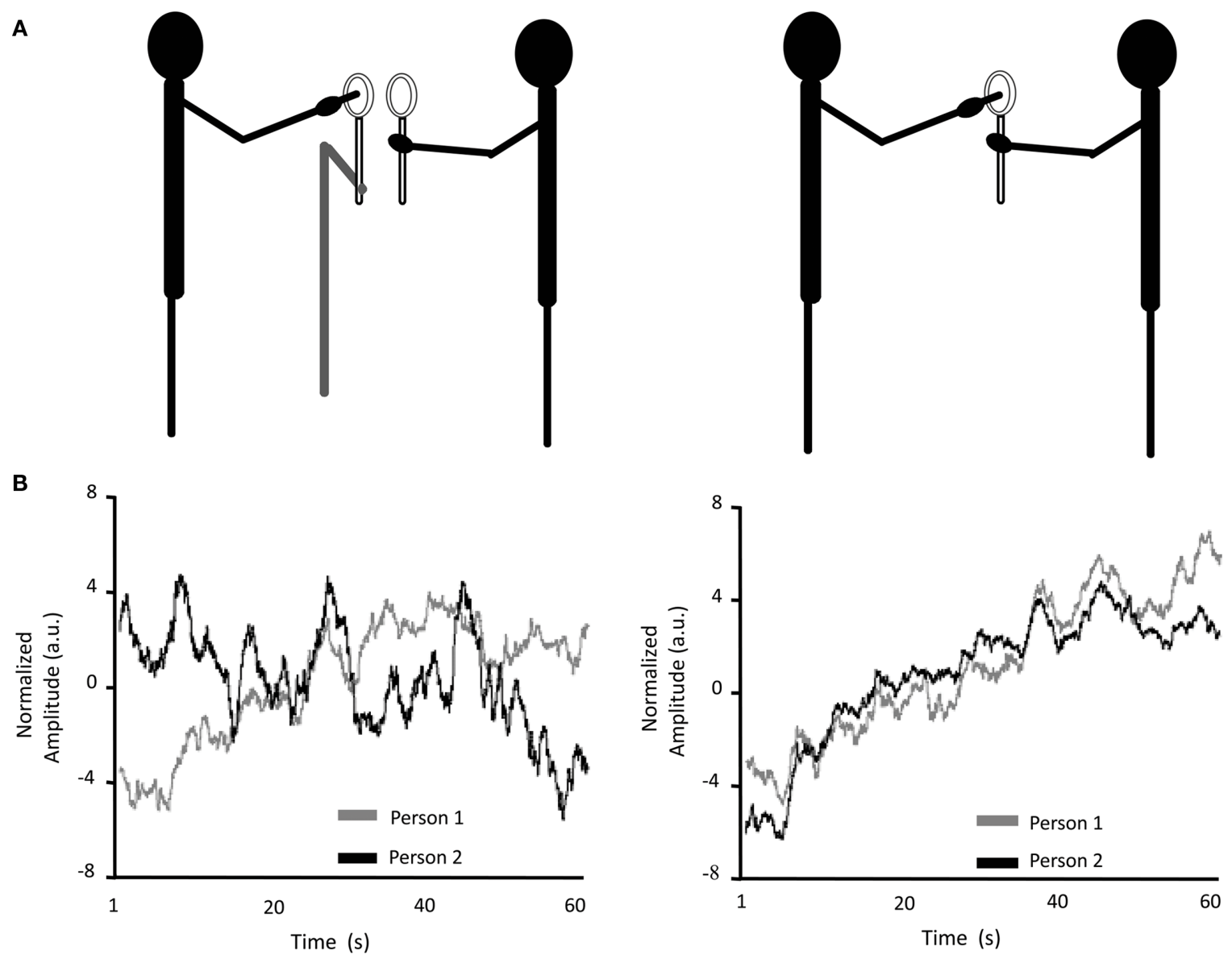

FIGURE 3 | (A) Depiction of the individual- (left) and interpersonal- (right) task conditions from Ramenzoni (2008). (B) Time series of the data projected onto the intrapersonal principle components from the individual (left) and interpersonal-

task (right) conditions. The striking coordination in the interpersonal-task condition was confirmed by cross-recurrence quantification analysis, which revealed a greater degree and higher stability of coupling in that condition.

Principal component analysis was first performed separately on each participant's data. Vectors consisting of 12 displacement time series ( 4 body segments $\times 3$ spatial dimensions) were submitted to PCA to identify the principal dimensions or modes of variation for each participant. These modes contained contributions of all the measured body segments in all spatial dimensions. For both individual- and interpersonal-task conditions six components were required to account for $90 \%$ of the variance in the original dataset - a two-fold dimensional reduction. We then projected each participant's movements onto the participant's first principal component, creating a new variable that expressed change over time along the dimension of primary variation (Figure 3B). We used CRQA to quantify the coordination between these new time series. CRQA revealed a significantly greater degree of coordination as well as significantly more stable coordination between the principal components for the interpersonal- than individual-task condition.

Data from both participants - a 24-dimensional vector $(2$ participants $\times 4$ body segments $\times 3$ spatial dimensions) - were submitted to a second PCA. For the individual-task condition six components accounted for $90 \%$ of the variance, but for the interpersonal-task condition there was greater dimensional reduction and just four components were required. Additionally, the first component accounted for significantly more variance in the interpersonal- than the individual-task condition.

These findings are consistent with the interpersonal synergy hypothesis. However, PCA cannot provide unequivocal evidence for the existence of synergies, because it does not directly measure reciprocal compensation (Latash, 2008).

\section{UCM ANALYSIS OF RHYTHMIC MOVEMENT COORDINATION}

Black et al. (2007) used the UCM approach to determine whether interpersonal coordination involves dimensional compression and reciprocal compensation. They had participants oscillate a handheld pendulum (Kugler and Turvey, 1987) in each hand (intrapersonal coordination) at a metronome-specified frequency to produce relative phase patterns corresponding to in-phase $\left(\phi \approx 0^{\circ}\right)$ or anti-phase $\left(\phi \approx 180^{\circ}\right)$, and had two participants, each holding a pendulum in one hand (interpersonal coordination), produce the same patterns. The mean and standard deviation of $\phi$ results conformed to HKB model predictions.

Black et al. (2007) treated $\phi$ as the performance variable (with desired values of $0^{\circ}$ or $180^{\circ}$ for in-phase and anti-phase, respectively), and the position and velocity of each hand as component DF. 
Those variables determine each hand's phase angle - the point of the oscillating hand in its ever-repeating movement cycle - and $\phi$ is the difference between the two hands' phase angles. The UCM analysis thus quantified how variation in the position and velocity of each hand was structured (or not) to preserve the goal $\phi$ value.

In all cases the ratio of compensated to uncompensated variance was $>1$, indicating the presence of synergies for intrapersonal and interpersonal coordination. Dimensional compression occurred because a lower dimensional variable, $\phi$, was selectively stabilized via reciprocal compensation among the components. Ratios were higher for intrapersonal (mean $=2.71$ ) than interpersonal (mean $=1.995)$ coordination, paralleling findings of greater coupling strength for intrapersonal coordination (Schmidt et al., 1998; Richardson et al., 2008b). The similitude between interpersonal and intrapersonal coordination was further reinforced because other manipulations, such as whether subjects were instructed to produce in-phase or anti-phase movement patterns, produced the same effects for interpersonal as for intrapersonal coordination [ratio was higher for in-phase (intrapersonal: 3.78; interpersonal: 2.31) than antiphase (intrapersonal: 1.64; interpersonal: 1.68)].

It could be argued that the existence of stable relative phase modes already provided evidence for synergies in interpersonal rhythmic coordination, at least insofar as this evidences dimensional compression. However, an observed stable value of $\phi$ is mute regarding whether $\phi$ is selectively stabilized through synergistic compensations. No specific value of $\phi$ identifies the existence of a synergy, and neither a change in the mean nor the standard deviation of $\phi$ indicates a change in synergy strength.

The Black et al. (2007) results provide direct evidence for interpersonal synergies. They were weaker than intrapersonal synergies, but nonetheless exhibited reciprocal compensation and dimensional compression. The findings suggest synergies are not hardwired features of an actor's neuromuscular system, but instead they are emergent properties of perception-action systems that are linked together informationally (in the case of interpersonal coordination, the informational linkage was visual).

\section{CONCLUSION}

The evidence reviewed here is consistent with claims that movement system DF residing in different actors are coupled to form low-dimensional, reciprocally compensating synergies. This may be controversial, because interpersonal synergies span organisms and extend beyond boundaries of skin, with the coupling among movement system DF achieved (in these studies) visually. It is difficult to envision how "control" might reside beyond the boundaries of an organism, or more specifically beyond a particular, singular component of an individual's brain. The control evident during interpersonal coordination may be an emergent property of a social perception-action system (Fowler et al., 2008; Richardson et al., 2008a, 2010; Schmidt and Richardson, 2008; Marsh et al., 2009; Shockley et al., 2009).

A key point to consider with regard to situating this approach scientifically and philosophically is that it emphasizes interactions and relations instead of fixed neuroanatomical components. This echoes the distinction between interaction-dominant dynamics and component-dominant dynamics described by Van Orden et al. (2003). In component-dominant dynamics, the emphasis is on the structure of fixed components. Component-dominant dynamics is assumed by classical cognitivism, with its emphasis on static modules that perform their functions in relative isolation of the activity of other modules. Interaction-dominant dynamics emphasizes interactions between components rather than their structure, entailing a functional, dynamical approach to understanding coordinated action. Instead of focusing on component structures, interaction-dominant dynamics focuses on "structures of process" (Juarrero, 1999, p. 124) that sustain patterns of organization. Interpersonal synergies might be prime examples of structures of process.

How might a structure of process be able to exert "control" over movements? This question raises another key point: The interpersonal synergy approach, with its roots in self-organizing complex systems, entails notions of "mechanism" and "causality" that are broader than the usual (Newtonian) sense of the terms as involving only efficient causes, the kinds of forceful interactions produced by colliding cogs or billiard balls (Rosen, 1991; Juarrero, 1999; Van Orden et al., 2005). Complex systems exhibit circular causality; bottom-up processes give rise to macroscopic patterns that simultaneously constrain the components from the top down. Constraints play the role of causal mechanisms in complex systems insomuch as constraints allow or deny certain states. Constraints limit the DF of a system, but do not cause the system to take on particular states by virtue of forceful "pushes" (local, efficient causes). Control (manipulation of the movement system) first entails coordination (organization of the movement system), as anticipated by Bernstein (1967).

Control parameters are constraints that guide the movement system through sequences of stable states (identified by values of order parameters). In rhythmic movement coordination, $\phi$ is the order parameter and movement frequency is a control parameter that, when varied, results in transitions between coordination patterns. Movement frequency has been identified as a control parameter for interpersonal rhythmic coordination (Schmidt et al., 1990), but the identification of control parameters for other interpersonal synergies remains an important avenue for future research. Possibilities include attention to movement information (Richardson et al., 2007; Schmidt et al., 2007), individual or social intentions or goals, perceived social differences, or rapport and liking (e.g., Chartrand and Bargh, 1999; Hove and Risen, 2009; Marsh et al., 2009; Miles et al., 2010), to name a few.

The alternative hypothesis to interpersonal synergies is that coordination observed between agents results because they execute similar but independent motor programs anchored in shared representations. Mirror neurons - neurons in the pre-motor cortex that are activated when observing others performing actions - suggest a common neural basis for motor control and action understanding. Watching someone engage in an action is hypothesized to activate the actor's own motor representation of that action, resulting in a shared motor representations. It is claimed that shared representations facilitate understanding others' actions (e.g., Blakemore and Decety, 2001; Richardson and Dale, 2005; Sebanz et al., 2006), which may explain why motor coordination occurs spontaneously in social contexts. This would not predict the synergistic nature of interpersonal coordination that we have described, however. Although this approach might predict dimensional compression 
(PCA would be sensitive to any correlations between the two actors' movements), it would not predict reciprocal compensation. Reciprocal compensation means that the actors' movements are not independent, directly contradicting this hypothesis.

Interpersonal coordination is a fundamental means of forming social units (Marsh et al., 2009). Interpersonal synergies may help build rapport (Bernieri et al., 1996; Chartrand and Bargh, 1999; Hove and Risen, 2009; Marsh et al., 2009). Being psychologically distanced from another individual can inhibit the emergence of interpersonal synergies (Miles et al., 2010). Interpersonal synergies facilitate memory for those with whom we interact (Miles et al., 2010) and can more generally facilitate performance of social cognitive or linguistic tasks (Richardson and Dale, 2005; Shockley et al., 2009). Synergies might also be a mechanism for interpersonal coordination in team sports (e.g., Passos et al., 2009). For example, highly effective teams might exhibit a greater

\section{REFERENCES}

Bernieri, F. J., and Rosenthal, R. (1991). "Interpersonal coordination: behavioural matching and interactional synchrony," in Fundamentals of Nonverbal Behavior, eds R.S. Feldman and B. Rime (Cambridge: Cambridge University Press), 401-432.

Bernieri, F. J., Gillis, J. S., Davis, J. M., and Grahe, J.E. (1996). Dyad rapport and the accuracy of its judgment across situations: a lens model analysis. J. Pers. Soc. Psychol. 71, 110-129.

Bernstein, N.A. (1967). Coordination and Regulation of Movements. New York: Pergamon Press.

Black, D. P., Riley, M. A., and McCord, C. K. (2007). Synergies in intra- and interpersonal interlimb rhythmic coordination. Motor Control 11, 348-373.

Blakemore, S. J., and Decety, J. (2001). From the perception of action to the understanding of intention. Nat. Rev. Neurosci. 2, 561-567.

Chartrand, T. L., and Bargh, J. A. (1999). The chameleon effect: the perceptionbehavior link and social interaction. J. Pers. Soc. Psychol. 76, 893-910.

Cohn, J. F., and Tronick, E. Z. (1988). Mother-infant face-to-face interaction: influence is bidirectional and unrelated to periodic cycles in either partner's behavior. Dev. Psychol. 24, 386-392.

Condon, W. S., and Ogston, W. D. (1967). A segmentation of behavior. J. Psychiatr. Res. 5, 221-235.

Condon, W. S., and Ogston, W. D. (1971). "Speech and body motion synchrony of the speaker-hearer," in The Perception of Language, eds D. Horton and J. Jenkins (Columbus, OH: Charles E. Merrill), 150-184.

Daffertshofer, A., Lamoth, C. J. C., Meijer, O. G., and Beek, P. J. (2004). PCA in studying coordination and variability: a tutorial. Clin. Biomech. 19, 415-428.
Forner-Cordero, A., Levin, O., Li, Y., and Swinnen, S. P. (2005). Principal component analysis of complex multijoint coordinative movements. Biol. Cybern. 93, 63-78.

Fowler, C. A., Richardson, M. J., Marsh, K., and Shockley, K. D. (2008). "Language use, coordination, and the emergence of cooperative action," in Coordination: Neural, Behavioral and Social Dynamics, eds A. Fuchs and V. Jirsa (Berlin: Springer-Verlag), 161-180.

Garrod, S., and Pickering, M. J. (2004). Why is conversation so easy? Trends Cogn. Sci. 8, 8-11. Joint action, interactive alignment, and dialog. Top. Cogn. Sci. 1, 292-304.

Gelfand, I. M., and Tsetlin, M. L. (1966). "On mathematical modeling of the mechanisms of the central nervous system," in Models of the StructuralFunctional Organization of Certain Biological Systems, eds I. M. Gelfand, V.S., Gurfinkel, S. V. Fomin, and M. L. Tsetlin (Moscow: Nauka), 9-26.

Haken, H. (1983). Synergetics: An Introduction. New York: Springer-Verlag.

Haken, H., Kelso, J. A. S., and Bunz, H. (1985). A theoretical model of phase transitions in human hand movements. Biol. Cybern. 51, 347-356.

Hove, M. J., and Risen, J. L. (2009). It's all in the timing: interpersonal synchrony increases affiliation. Soc. Cogn. 27, 949-961.

Juarrero, A. (1999). Dynamics in Action. Cambridge, MA: MIT Press.

Kay, B. A. (1988). The dimensionality of movement trajectories and the degrees of freedom problem: a tutorial. Hum. Mov. Sci. 7, 343-364.

Kay, B. A., Kelso, J. A. S., Saltzman, E. L., and Schöner, G. (1987). Space-time behavior of single and bimanual rhythmical movements: data and
Garrod, S., and Pickering, M. J. (2009).

degree of reciprocal compensation than poorly effective ones. Developing a detailed understanding of interpersonal synergies may have broad and significant implications for understanding social perception-action systems.

The interpersonal synergy hypothesis can be subjected to additional tests, using a range of paradigms and complementary methods such as the UCM analysis and PCA. This may be a useful agenda for future research. If the hypothesis is supported movement science will be charged with the important task of broadening its conceptions of fundamental processes of coordination and control, and areas such as social psychology or sports psychology might benefit from the development of new tools and paradigms.

\section{ACKNOWLEDGMENT}

Supported by National Science Foundation grants BCS 0926662 and DHB 0728743.

limit cycle model. J. Exp. Psychol. Hum. Percept. Perform. 13, 178-192.

Kelso, J. A. S. (1994). "Elementary coordination dynamics," in Interlimb Coordination: Neural, Dynamical, and Cognitive Constraints, eds S. P. Swinnen, H. Heuer, J. Massion, and P. Casaer (New York: Academic Press), 301-318.

Kelso, J. A. S. (1995). Dynamic Patterns: The Self-Organization of Brain and Behavior. Cambridge, MA: MIT Press.

Kelso, J. A. S., Tuller, B., VatikiotisBateson, E., and Fowler, C. A. (1984). Functionally specific articulatory cooperation following jaw perturbations during speech: evidence for coordinative structures. J. Exp. Psychol. Hum. Percept. Perform. 10, 812-832.

Kendon, A. (1970). Movement coordination in social interaction: some examples described. Acta Psychol. 32, 101-125.

Kugler, P. N., Kelso, J. A. S., and Turvey, M. T. (1980). "On the concept of coordinative structures as dissipative structures: I. Theoretical lines of convergence," in Tutorials in Motor Behavior, eds G. E. Stelmach and J. Requin (Amsterdam: North-Holland), 3-47.

Kugler, P. N., and Turvey, M. T. (1987). Information, Natural Law, and the Self-Assembly of Rhythmic Movement. Hillsdale, NJ: Erlbaum.

Latash, M. L. (2008). Synergy. New York: Oxford University Press.

Latash, M. L., Scholz, J. P., and Schöner, G. (2002). Motor control strategies revealed in the structure of motor variability. Exerc. Sport Sci. Rev. 30, 26-31.

Marsh, K. L., Richardson, M. J., Baron, R. M., and Schmidt, R. C. (2006). Contrasting approaches to perceiving and acting with others. Ecol. Psychol. $18,1-38$.

Marsh, K. L., Richardson, M. J., and Schmidt, R. C. (2009). Social connec- tion through joint action and interpersonal coordination. Top. Cogn. Sci. 1, 320-339.

Miles, L. K., Griffiths, J. L., Richardson, M. J., and Macrae, C. N. (2010). Too late to coordinate: contextual influences on behavioral synchrony. J. Exp. Soc. Psychol. 40, 52-60.

Newtson, D., Enquist, G., and Bois, J. (1977). The objective basis of behavior units. J. Pers. Soc. Psychol. 35, 847-862.

Newtson, D., Hairfield, J., Bloomingdale, J., and Cutino, S. (1987). The structure of action and interaction. Soc. Cogn. 5, 191-237.

Nicolis, G., and Prigogine, I. (1977). Self-Organization in Nonequilibrium Systems. New York: John Wiley \& Sons.

Passos, P., Araújo, D., Davids, K., Gouveia, Serpa, S., Milho, J., and Fonseca, S. (2009). Interpersonal pattern dynamics and adaptive behavior in multiagent neurobiological systems: conceptual model and data. J. Mot. Behav. 41, 445-459.

Ramenzoni, V. C. (2008). Effects of Joint Task Performance on Interpersonal Postural Coordination. Unpublished doctoral dissertation, University of Cincinnati, Cincinnati, $\mathrm{OH}$.

Reed, K., Peshkin, M., Hartmann, M. J., Grabowecky, M., Patton, J., and Vishton, P. M. (2006). Haptically linked dyads: are two motor control systems better than one? Psychol. Sci. 17, 365-366.

Richardson, D. C., and Dale, R. (2005). Looking to understand: the coupling between speakers' and listeners' eye movements and its relationship to discourse comprehension. Cogn. Sci. 29, 1045-1060.

Richardson, D. C., Dale, R., and Shockley, K. (2008a). "Synchrony and swing in conversation: coordination, temporal dynamics, and communication," in Embodied Communication in Humans 
and Machines, eds I. Wachsmuth, M. Lenzen, and G. Knoblich (Oxford: Oxford University Press), 75-93.

Richardson, M. J., Lopresti-Goodman, S., Mancini, M., Kay, B. A., and Schmidt, R. C. (2008b). Comparing the attractor strength of intra- and interpersonal interlimb coordination using cross recurrence analysis. Neurosci. Lett. 438, 340-345.

Richardson, M. J., Marsh, K. L., and Schmidt, R. C. (2010). "Challenging egocentric notions of perceiving, acting, and knowing," in The Mind in Context, eds L. F. Barrett, B. Mesquita, and E. Smith (New York: Guilford), 307-333.

Richardson, M. J., Schmidt, R. C., and Kay, B. A. (2007). Distinguishing the noise and attractor strength of coordinated limb movements using recurrence analysis. Biol. Cybern. 96, 59-78.

Riley, M. A., and Turvey, M. T. (2002). Variability and determinism in motor behavior. J. Mot. Behav. 34, 99-125.

Rosen, R. (1991). Life Itself. New York: Columbia University Press.

Schmidt, R. C., Bienvenu, M., Fitzpatrick, P. A., and Amazeen, P. G. (1998). A comparison of intra- and interpersonal coordination: coordination breakdowns and coupling strength. J. Exp. Psychol. Hum. Percept. Perform. 24, 884-900.

Schmidt, R. C., Carello, C., and Turvey, M. T. (1990). Phase transitions and critical fluctuations in the visual coordination of rhythmic movements between people. J. Exp. Psychol. Hum. Percept. Perform. 16, 227-247.
Schmidt, R. C., and Richardson, M. J. (2008). "Dynamics of interpersonal coordination," in Coordination: Neural, Behavioral and Social Dynamics, eds A. Fuchs and V. K. Jirsa (Berlin: SpringerVerlag), 281-307.

Schmidt, R. C., Richardson, M. J., Arsenault, C., and Galantucci, B. (2007). Unintentional entrainment to an environmental rhythm: effect of eye tracking. J. Exp. Psychol. Hum. Percept. Perform. 33, 860-870.

Schmidt, R. C., and Turvey, M. T. (1994). Phase-entrainment dynamics of visually coupled rhythmic movements. Biol. Cybern. 70, 369-376.

Scholz, J. P., and Schöner, G. (1999). The uncontrolled manifold concept: identifying control variables for a functional task. Exp. Brain Res. 126, 289-306.

Sebanz, N., Bekkering, H., and Knoblich, G. (2006). Joint action: bodies and minds moving together. Trends Cogn. Sci. 10, 70-76.

Shockley, K. (2005). "Cross recurrence quantification of interpersonal postural activity." in Tutorials in Contemporary Nonlinear Methods for the Behavioral Sciences, eds M. A. Riley and G. Van Orden, 142-177. Available at: http://www.nsf.gov/sbe/bcs/pac/ $\mathrm{nmbs} / \mathrm{nmbs}$.jsp

Shockley, K., Butwill, M., Zbilut, J. P., and Webber, C. L. Jr. (2002). Cross recurrence quantification of coupled oscillators. Phys. Lett. A 305, 59-69.

Shockley, K., Richardson, D. C., and Dale, R. (2009). Conversation and coordinative structures. Top. Cogn. Sci. 1, 305-319.
Shockley, K., Santana, M. V., and Fowler, C. A. (2003). Mutual interpersonal postural constraints are involved in cooperative conversation. J. Exp. Psychol. Hum. Percept. Perform. 29, 326-332.

Tognoli, E., Lagarde, J., DeGuzman, C. and Kelso, J. A. S. (2007). The phi complex as a neuromarker of human social coordination. Proc. Natl. Acad. Sci. U.S.A. 104, 8190-8195.

Tuller, B., Turvey, M. T., and Fitch, H. F. (1982). "The Bernstein perspective: II. The concept of muscle linkage or coordinative structure," in Human Motor Behavior: An Introduction, ed J. A. S. Kelso (Hillsdale, NJ: Lawrence Erlbaum Associates), 253-270.

Turvey, M. T. (1990). Coordination. Am. Psychol. 45, 938-953.

Turvey, M. T., and Carello, C. (1996) "Dynamics of Bernstein's levels of synergies," in Dexterity and It Development, eds M. L. Latash and M. T. Turvey (Mahwah, NJ: Erlbaum), 339-377.

Turvey, M. T., Fitch, H. F., and Tuller, B. (1982). "The Bernstein perspective: 1 . The problems of degrees of freedom and context-conditioned variability," in Human Motor Behavior: An Introduction, ed. J. A. S. Kelso (Hillsdale, NJ: Lawrence Erlbaum Associates), 239-252.

Turvey, M. T., Shaw, R. E., and Mace, W. (1978). "Issues in the theory of action: degrees of freedom, coordinative structures, and coalitions," in Attention and Performance VII, ed. J. Requin (Hillsdale, NJ: Lawrence Erlbaum Associates), 557-595.
Van Orden, G. C., Holden, J. G., and Turvey, M.T.(2003).Self-organization of cognitive performance. J. Exp. Psychol. Gen. 132, 331-350.

Van Orden, G. C., Holden, J. G., and Turvey, M. T. (2005). Human cognition and $1 / f$ scaling. J. Exp. Psychol. Gen. 134, 117-123.

Webber, C. L. Jr., and Zbilut, J. P. (1994). Dynamical assessment of physiological systems and states using recurrence plot strategies. J. Appl. Physiol. 76, 965-973.

Conflict of Interest Statement: The authors declare that the research was conducted in the absence of any commercial or financial relationships that could be construed as a potential conflict of interest.

Received: 22 September 2010; paper pending published: 01 November 2010; accepted: 23 February 2011; published online: 09 March 2011.

Citation: Riley MA, Richardson MJ Shockley K and Ramenzoni VC (2011) Interpersonal synergies. Front. Psychology 2:38. doi: 10.3389/fpsyg.2011.00038

This article was submitted to Frontiers in Movement Science and Sport Psychology, a specialty of Frontiers in Psychology. Copyright (C) 2011 Riley, Richardson Shockley and Ramenzoni. This is an open-access article subject to an exclusive license agreement between the authors and Frontiers Media SA, which permits unrestricted use, distribution, and reproduction in any medium, provided the original authors and source are credited. 